\title{
Equivalence principle and electromagnetic field: no birefringence, no dilaton, and no axion
}

\author{
Friedrich W. Hehl* \\ Institute for Theoretical Physics, University of Cologne, 50923 Köln, Germany \\ Yuri N. Obukhovț \\ Institute for Theoretical Physics, University of Cologne, 50923 Köln, Germany
}

(Dated: 16 May 2007, file equiv07.tex)

\begin{abstract}
The coupling of the electromagnetic field to gravity is discussed. In the premetric axiomatic approach based on the experimentally well established conservation laws of electric charge and magnetic flux, the Maxwell equations are the same irrespective of the presence or absence of gravity. In this sense, one can say that the charge "substratum" and the flux "substratum" are not influenced by the gravitational field directly. However, the interrelation between these fundamental substrata, formalized as the spacetime relation $H=H(F)$ between the 2-forms of the electromagnetic excitation $H$ and the electromagnetic field strength $F$, is affected by gravity. Thus the validity of the equivalence principle for electromagnetism depends on the form of the spacetime relation. We discuss the nonlocal and local linear constitutive relations and demonstrate that the spacetime metric can be accompanied also by skewon, dilaton, and axion fields. All these premetric companions of the metric may eventually lead to a violation of the equivalence principle.

PACS numbers: 04.20.Cv; 04.80.Cc; 03.50.De; 41.20.Jb

Keywords: Equivalence principle, classical electrodynamics, general relativity, skewon field, dilaton field, axion field

*Electronic address: hehl@thp.uni-koeln.de

$\dagger$ Also at: Department of Physics \& Astronomy, University of Missouri-Columbia, Columbia, MO 65211, USA

${ }^{\ddagger}$ Electronic address: yo@thp.uni-koeln.de

$\S$ Also at: Department of Theoretical Physics, Moscow State University, 117234 Moscow, Russia
\end{abstract}




\section{INTRODUCTION}

In the modern theory of gravity, one usually distinguishes three forms of the equivalence principle. Namely, the weak equivalence principle, relying on the experimental fact of the equality of the gravitational and inertial masses, states that all massive objects (test bodies) with the same initial velocity follow the same trajectories in a gravitational field (the universality of a free fall). The Einsteinian equivalence principle postulates that the result of any local non-gravitational experiment in a local inertial frame ("Einstein's elevator") is the same everywhere in spacetime. A generalization of this statement, when the same is true for any local experiment, including the gravitational ones, is called the strong equivalence principle.

In gravitational theory, the question of whether the equivalence principle is valid and which form of it (as well as the interrelations between these forms), belongs to the most fundamental issues. Theoretical analyses as well as experimental tests are conducted by many research groups. Generally speaking, the equivalence principle specifies the coupling of gravity to matter. Here we will not discuss all the aspects of this subject, but we will confine our attention to the coupling between gravity and electromagnetism.

This paper is dedicated to our friend and colleague Bahram Mashhoon on the occasion of his 60th birthday. One of us had quite often discussions with Bahram on physics topics for over more than 30 years, and he is very grateful to Bahram for sharing with him his profound insight into physics and astrophysics.

\section{ELECTROMAGNETISM AND EQUIVALENCE PRINCIPLE}

Our world is made, to a great extent, of electromagnetically interacting particles. Moreover, practically all the information about the near and the distant space comes to us in the form of electromagnetic waves. Thus it is very important to verify whether the equivalence principle is valid for electromagnetically interacting systems of fields and particles. In the simplest formulation, we can ask: Does a photon move, since it is massless, along a null geodesic line in a curved spacetime, in accordance with the equivalence principle?

Within the framework of general relativity, a light ray can be extracted from classical electrodynamics in its geometrical optics limit, i.e., for wavelengths much smaller than the 
local curvature radius of space. Accordingly, the bending of light can be understood as a result of a nontrivial refractive index of spacetime due to the coupling of the electromagnetic field $F$ to the gravitational field $g$, see Skrotskii et al. [1, 2]. Classically, we have in nature just these two fundamental fields $F$ and $g$, the weak and the strong fields being confined to microphysical dimensions of $10^{-20} \mathrm{~m}$ or $10^{-15} \mathrm{~m}$, respectively. Therefore, the coupling of $F$ and $g$ is of foremost importance in classical physics.

\section{PREMETRIC FORMULATION OF ELECTRODYNAMICS}

The equivalence principle is about the coupling of matter to gravity. In order to understand the coupling of electromagnetism to gravity, it is most convenient and reasonable to start from the premetric formulation of the electrodynamical theory. Following the early work of Kottler, Cartan, and van Dantzig, one can indeed develop an axiomatic approach to the electromagnetic field [3, 4, 5] without assuming any specific geometric structure beyond the differentiable structure of the spacetime manifold, see also [6, 7]. At the heart of this approach, there are the well established experimental facts (that can be formulated as fundamental axioms) of electric charge and magnetic flux conservation. The corresponding definitions and equations can be written down very succinctly in the calculus of exterior differential forms (Cartan calculus). Charge and flux conservation gives rise to the two basic objects of the electromagnetic theory, the electromagnetic excitation 2-form $H$ (with twist, or odd) and the electromagnetic field strength 2-form $F$ (without twist, or even), and it ultimately yields, with the electric current $J$, the field equations

$$
d H=J, \quad d F=0,
$$

with

$$
d J=0
$$

The set (1) represents the Maxwell equations. They are independent of metric and connection. At this stage, the equivalence principle looks empty: Since the Maxwell equations (1) are formulated in a coordinate and frame independent way, they are valid in this form in arbitrary coordinate systems and frames, be it in a flat or in a curved spacetime. 


\section{SPACETIME (VACUUM CONSTITUTIVE) RELATION AND EQUIVA- LENCE PRINCIPLE}

In order to make electrodynamics a predictive theory, we need to complete Maxwell's equations (1) with the spacetime relation (or vacuum constitutive relation). The latter links the excitation to the field strength,

$$
H=H(F) .
$$

Only the constitutive relation "feels", up to a conformal factor, the presence of a flat or a nonflat metric $g$, i.e., the constitutive relation couples to the conformally invariant part of the metric. In addition, premetric companions can come into play through the spacetime relation (3), as we will see later. The coupling of electromagnetism to gravity becomes almost trivial in this sense. In plain words, the test of the equivalence principle for electromagnetically interacting systems amounts to the test of the spacetime relation (3).

The spacetime relation can be quite non-trivial, in general. For example, it can be nonlinear and even nonlocal. The nonlinearity can be fundamental or effective, see the discussion in [3]. Here we will limit our attention to the linear spacetime relations.

\section{A. Nonlocal, linear: Volterra-Mashhoon}

Let us choose arbitrary local spacetime coordinates $x^{i}$ and the local coframe field $\vartheta^{\alpha}$. The latter is usually determined by specifying the physical observers and their local reference frames that they use for the making of physical (in particular electrodynamical) measurements. Then we have with respect to the coframe $\vartheta^{\alpha}=e_{i}{ }^{\alpha} d x^{i}$ for an in general non-inertial observer

$$
H=\frac{1}{2} H_{\alpha \beta} \vartheta^{\alpha} \wedge \vartheta^{\beta}, \quad F=\frac{1}{2} F_{\alpha \beta} \vartheta^{\alpha} \wedge \vartheta^{\beta} .
$$

Generalizing the locality assumption, Mashhoon [8] postulated, following similar suggestions of Volterra, the nonlocal constitutive law

$$
H_{\alpha \beta}(\tau, \xi)=\frac{1}{2} \int d \tau^{\prime} K_{\alpha \beta}^{\gamma \delta}\left(\tau, \tau^{\prime}\right) F_{\gamma \delta}\left(\tau^{\prime}, \xi\right) .
$$

The response kernel in (5) is defined by the acceleration and rotation of the observer's reference system. It is a constitutive law for the vacuum as viewed from a non-inertial frame 
of reference. The general form of the kernel can be worked out explicitly, see [3], e.g., $(u$ is the observer's 4-velocity):

$$
\left.K_{\alpha \beta}^{\gamma \delta}\left(\tau, \tau^{\prime}\right)=\frac{1}{2} \epsilon_{\alpha \beta}{ }^{\lambda[\delta}\left(\delta_{\lambda}^{\gamma]} \delta\left(\tau-\tau^{\prime}\right)-u\right\rfloor \Gamma_{\lambda}^{\gamma]}\left(\tau^{\prime}\right)\right)
$$

The influence of non-inertiality is manifest in the presence of the connection 1 -form $\Gamma_{\alpha}{ }^{\beta}$. It has been shown that this kernel is the only consistent one, see the review [9]. So far, there is no experimental support for (5) and (6) in the case of vacuum electrodynamics.

\section{B. Local, linear}

A very important case is that of a local and homogeneous linear constitutive law between the components of the two-forms $H$ and $F$. In local coordinates, we have $H=\frac{1}{2} H_{i j} d x^{i} \wedge$

$d x^{j}, F=\frac{1}{2} F_{i j} d x^{i} \wedge d x^{j}$. The linear spacetime relation then postulates the existence of a constitutive tensor with $6 \times 6=36$ components $\kappa_{i j}{ }^{k l}(t, x)=-\kappa_{j i}{ }^{k l}=-\kappa_{i j}{ }^{l k}$ such that

$$
H_{i j}=\frac{1}{2} \kappa_{i j}^{k l} F_{k l} \text {. }
$$

This kind of an ansatz we know from the physics of anisotropic crystals. Taking the LeviCivita symbol, we can introduce the alternative constitutive tensor density by

$$
\chi^{i j k l}:=\frac{1}{2} \epsilon^{i j m n} \kappa_{m n}^{k l}, \quad \text { or } \quad \kappa_{i j}^{k l}=\frac{1}{2} \epsilon_{i j m n} \chi^{m n k l} .
$$

With the linear constitutive law (as with more general laws), we can, in the case of vanishing dissipative effects, set up a Lagrangian 4-form; here we call it $V_{\text {lin. }}$. Because of $H=-\partial V_{\text {lin }} / \partial F$, the Lagrangian must then be quadratic in $F$. Thus we find

$$
V_{\text {lin }}=-\frac{1}{2} H \wedge F=-\frac{1}{8} \chi^{i j k l} F_{i j} F_{k l} d x^{0} \wedge d x^{1} \wedge d x^{2} \wedge d x^{3} .
$$

The components of the field strength $F$ enter in a symmetric way. Therefore, within the framework of the variational approach, we can impose the symmetry condition $\chi^{i j k l}=\chi^{k l i j}$ on the constitutive tensor thus reducing the number of its independent components to 21 at this stage. The components $\kappa_{i j}{ }^{k l}$ carry the dimension of $[\kappa]=[\chi]=q^{2} / \mathfrak{h}=1 /$ resistance. We denote the dimension of charge by $q$ and the dimension of action by $\mathfrak{h}$.

In this way, we come naturally to the so-called $\chi-g$ scheme proposed by $\mathrm{Ni}[10,11,12,13$ ] as a general phenomenological framework for the discussion of the equivalence principle. It includes narrower schemes such as [14, 15, 16], e.g.. 


\section{WAVE PROPAGATION}

How does an electromagnetic wave (a "photon") propagate in space and time?

\section{A. Generalized Fresnel equation}

In the geometric optics approximation (equivalently, in the Hadamard approach) an electromagnetic wave is described by the propagation of a discontinuity of the electromagnetic field [17]. The surface of discontinuity $S$ is defined locally by a function $\Phi$ such that $\Phi=$ const on $S$. The wave covector $q:=d \Phi$ contains the essential information about the propagation of electromagnetic waves ("light"). We define the 4th-order Tamm-Rubilar (TR) tensor density of weight +1 ,

$$
\mathcal{G}^{i j k l}(\chi):=\frac{1}{4 !} \epsilon_{m n p q} \epsilon_{r s t u} \chi^{m n r(i} \chi^{j|p s| k} \chi^{l) q t u} .
$$

It is totally symmetric $\mathcal{G}^{i j k l}(\chi)=\mathcal{G}^{(i j k l)}(\chi)$. Thus, it has 35 independent components. One can demonstrate that the wave covectors satisfy the extended Fresnel equation that is generally covariant in 4 dimensions:

$$
\mathcal{G}^{i j k l}(\chi) q_{i} q_{j} q_{k} q_{l}=0
$$

In other words, the test of the equivalence principle for electromagnetic waves reduces to the investigation of the structure of the wave surface (11). The latter is determined solely by the spacetime relation, i.e., by the constitutive tensor (8) that enters the TR tensor (10). New derivations of the extended Fresnel equation have been given recently by Itin [20] and Perlick [21].

\section{B. Birefringence, skewon}

In general, the wave covectors $q$ lie on a quartic Fresnel wave surface, not exactly what we are observing in vacuum at the present epoch of our universe. One can gain further insight into the wave propagation by decomposing the constitutive tensor into the three irreducible parts [18, 19]

$$
\begin{aligned}
\kappa_{i j}{ }^{k l} & ={ }^{(1)} \kappa_{i j}{ }^{k l}+{ }^{(2)} \kappa_{i j}{ }^{k l}+{ }^{(3)} \kappa_{i j}{ }^{k l} \\
& ={ }^{(1)} \kappa_{i j}{ }^{k l}-4 \not_{[i}{ }^{[k} \delta_{j]}^{l]}+2 \alpha \delta_{[i}^{k} \delta_{j]}^{l} .
\end{aligned}
$$


The skewon and the axion fields are here conventionally defined by

$$
\oiint_{i}{ }^{j}=-\frac{1}{2} \kappa_{i k}{ }^{j k}+\frac{1}{8} \kappa \delta_{i}^{j}, \quad \alpha=\frac{1}{12} \kappa=\frac{1}{12} \kappa_{i j}{ }^{i j} .
$$

The principal (or the metric-dilaton) part ${ }^{(1)} \kappa_{i j}{ }^{k l}$ of the constitutive tensor with 20 independent components will eventually be expressed in terms of the metric (thereby cutting the 20 components in half).

It is straightforward to see that the axion $\alpha$ does not contribute to the extended Fresnel equation at all (although they can produce effects beyond the geometric optics approximation [22, 23]), whereas for a vanishing principal part ${ }^{(1)} \kappa_{i j}{ }^{k l}=0$, there is no wave propagation. In general, a nonvanishing skewon yields a very complicated picture for the wave surface [24], i.e., a highly nontrivial birefringent wave propagation.

One can prove [25] that by requiring the vanishing of birefringence, the quartic Fresnel wave surface reduces to a unique light cone. The latter defines, up to an overall conformal factor, a metric tensor $g^{i j}(x)$ with the correct Lorentzian signature, which is constructed directly in terms of the constitutive tensor $\kappa_{i j}{ }^{k l}$.

\section{Dilaton and axion}

Introducing the (Levi-Civita) dual of the excitation, $\check{H}^{i j}:=\frac{1}{2} \epsilon^{i j k l} H_{k l}$, we can finally rewrite the spacetime relation for vanishing birefringence in vacuum as

$$
\check{H}^{i j}=[\underbrace{\lambda(x)}_{\text {dilaton }} \sqrt{-g} g^{i k}(x) g^{j l}(x)+\underbrace{\alpha(x)}_{\text {axion }} \epsilon^{i j k l}] F_{k l},
$$

that is, we are left with the constitutive fields dilaton $\lambda$, metric $g^{i j}$, and axion $\alpha$. The combination $\sqrt{-g} g^{i[k}(x) g^{l] j}(x)$ is conformally invariant. In exterior calculus, (14) reads

$$
H=\lambda(x)^{\star} F+\alpha(x) F,
$$

with the Hodge star operator *. In turn, the Lagrangian of the electromagnetic theory, including dilaton and axion, reads

$$
V=-\frac{1}{2}\left(\lambda F \wedge{ }^{\star} F+\alpha F \wedge F\right) .
$$




\section{Light cone: null geodesics for a photon}

Inserting the spacetime relation (14) into (10), we find that the quartic Fresnel surface

(11) reduces to the unique light cone, $g^{i j} q_{i} q_{j}=0$. By taking a covariant derivative of the latter, we have

$$
q^{i} \nabla_{k} q_{i}=q^{i} \nabla_{i} q_{k}=0
$$

where we used the fact that $\nabla_{[i} q_{j]}=\partial_{[i} \partial_{j]} \Phi \equiv 0$. Thus, we verified that the photon propagates along a null geodesic (17), in accordance with the equivalence principle.

\section{EXPERIMENTAL TESTS}

\section{A. No birefringence}

In vacuum, the propagation of light does not reveal any evidence of birefringence [26, 27]. Hence, one can conclude that, up to a certain limit, there is no skewon field, and the principal part of the constitutive tensor reduces to the standard Maxwell-Lorentz form. The detailed analysis of the experimental limits on the birefringence in vacuum can be found in [22, 28].

\section{B. No axion?}

As yet, the Abelian axion has not been found experimentally [29, 30, 31, 32, 33, 34], see also the discussion of Cooper and Stedman 35] on corresponding ring laser experiments. However, the recent PVLAS experiments [36, 37, 38] pointed to some optical activity of the vacuum provided an external magnetic field was present. A nontrivial axion field is considered as one of the possible explanations of these observations. However, the interpretation of these experiments is still under discussion and the situation is not completely clear. Probably more independent experiments are needed.

\section{No dilaton?}

There is no direct evidence of the dilaton as yet, i.e., under normal circumstances, the dilaton seems to be a constant field and thereby sets a certain scale, i.e., $\lambda(x)=\lambda_{0}$, where $\lambda_{0}$ is the admittance of free space the value of which is, in SI-units, $\lambda_{0} \cong 1 /(377 \Omega)$. 
However, the possibility of time and space variations of the fundamental constants is discussed in the literature both from an experimental and a theoretical point of view. Of particular interest are certain indications that the fine structure constant may slowly change on a cosmological time scale. $\mathrm{Ni}[12]$ points out that the variation of fundamental constants can be viewed as an indication of the violation of Einstein's equivalence principle.

Maxwell's equations follow from charge and flux conservation. Any charge is proportional to the elementary charge $e$, any flux proportional to the elementary flux $h / e^{2}$. Consequently, if the electron charge $e$ and the Planck constant $h$ keep their values constant (independent of time, e.g.), then the quantities proportional to them, or any power of them, namely $e^{n_{1}} h^{n_{2}}$, with $n_{1}$ and $n_{2}$ as integer numbers, are also conserved. Therefore the time independence of $e$ and $h$ are the raisons d'être of the Maxwell equations. Or the other way round: If we want to uphold the Maxwell equations and thus QED, then we have to demand $e=$ const and $h=$ const.

However, there is a different possibility. In order to recognize this, let us have a closer look at the definition of the fine structure constant:

$$
\alpha_{\mathrm{f}}=\frac{e^{2}}{2 \varepsilon_{0} c h}=\frac{e^{2}}{2 h \lambda_{0}}=\frac{\Omega_{0}}{2 R_{\mathrm{K}}}
$$

As we can see, the fine structure constant is explicitly given in terms of the ratio of two resistances - vacuum impedance $\Omega_{0}=1 / \lambda_{0}$ and von Klitzing constant $R_{\mathrm{K}}$ (the quantum Hall resistance). This relation is valid in all systems of units. Note that the speed of light c disappeared completely! Its "presence" in the first equality is in fact misleading (it is multiplied by $\varepsilon_{0}$ !) and the proper understanding is suggested only in the second equality where $\lambda_{0}$ shows up instead, together with $e$ and $h$.

In other words, the formula (18) demonstrates that of the two fundamental constants $\Omega_{0}$ and $c$ of electrodynamics, which appear naturally in the Maxwell-Lorentz electrodynamics (see the previous section), it is the vacuum impedance $\Omega_{0}$ which enters the fine structure constant and not the speed of light.

Recall now the argument [39] that $e$ and $h$, being 4D scalars, should not change in time and space provided one wants to uphold the validity of the Maxwell equations (1). Then the variation of the fine structure constant $\alpha_{\mathrm{f}}=\alpha_{\mathrm{f}}(t)$ forces us to conclude that $\lambda=\lambda(t)$. An inspection of the Maxwell Lagrangian (16) then shows that $\lambda$ becomes a dynamical dilaton field. In the axiomatic premetric approach to electrodynamics, we have a scalar and 
a pseudoscalar part of the spacetime relation that are independent of the spacetime metric: these are the dilaton $\lambda$ and the axion $\alpha$. The variability of the fine structure constant thus may be explained without changing the Maxwell equations and QED by the presence of the dilaton field in a generalized Maxwell-Lorentz spacetime relation.

\section{CONCLUSIONS}

We demonstrated here that the premetric axiomatic approach to electromagnetic theory provides a natural framework for the test of the equivalence principle. Which particular constitutive (spacetime) relation $H=H(F)$ is valid? This is the central question. A complete analysis of this question can be performed for the case of the local linear spacetime relation, for which an extended Fresnel equation (11) is derived in terms of the constitutive tensor $\kappa$. At present, there is no experimental evidence for the existence of birefringence for electromagnetic waves in vacuum. This fact alone reduces the structure of the constitutive tensor to the simple form (14) (or, equivalently, to the spacetime relation (15)), introducing the (optical) spacetime metric $g$ and its scalar and pseudoscalar companions, dilaton and axion. A possible indication that the two latter premetric fields are nontrivial, in particular a dilaton $\lambda$, can come from the possible variation of the fundamental physical constants. As to the axion, when $\alpha=$ const, it does not affect the wave propagation. However, as pointed

out in [13, 40], one may observe measurable effects at the interfaces between spacetime domains with different constant values of $\alpha$.

Acknowledgments. This work was done with the support of the DFG (Project He 528/21-1), Bonn.

[1] G.V. Skrotskii, The influence of gravitation on the propagation of light, Sov. Phys. Doklady 2 (1957) 226-229.

[2] A.M. Volkov, A.A. Izmest'ev, and G.V. Skrotskii, The propagation of electromagnetic waves in a Riemannian space, Sov. Phys. JETP 32 (1971) 686-689 [ZhETF 59 (1970) 1254-1261 (in Russian)].

[3] F.W. Hehl and Yu.N. Obukhov, Foundations of classical electrodynamics: Charge, flux, and metric (Birkhäuser: Boston, 2003) Progress in Mathematical Physics, vol. 33, 430 pp. 
[4] F.W. Hehl, Y. Itin, and Yu.N. Obukhov, Recent developments in premetric electrodynamics, in: Proceedings of the 3rd Summer School in Modern Mathematical Physics, 20-31 August 2004, Zlatibor, Serbia and Montenegro, Eds. B. Dragovich et al. (Institute of Physics: Belgrade, 2005) pp. 375-408.

[5] F.W. Hehl and Yu.N. Obukhov, Spacetime metric from local and linear electrodynamics: A new axiomatic scheme, in: "Special relativity: Will it survive the next 101 years?", Eds. J. Ehlers and C. Lämmerzahl, Lect. Notes in Phys. 702 (Springer: Berlin, 2006) 163-187.

[6] R.A. Puntigam, C. Lämmerzahl, and F.W. Hehl, Maxwell's theory on a post-Riemannian spacetime and the equivalence principle, Class. Quantum Grav. 14 (1997) 1347-1356.

[7] F.W. Hehl and Yu.N. Obukhov, How does the electromagnetic field couple to gravity, in particular to metric, nonmetricity, torsion, and curvature?, in: "Gyros, Clocks, Interferometers...: Testing Relativistic Gravity in Space”, Eds. C. Lämmerzahl, C.W.F. Everitt, and F.W. Hehl, Lect. Notes in Phys. 562 (Springer: Berlin, 2001) 479-504.

[8] B. Mashhoon, Nonlocal electrodynamics, in: Cosmology and Gravitation, Proc. VII Brasilian School of Cosmology and Gravitation, Rio de Janeiro, August 1993, M. Novello, editor. Editions Frontieres, Gif-sur-Yvette (1994) pp. 245-295.

[9] B. Mashhoon, Nonlocal electrodynamics of accelerated systems, E-print: hep-th/0702074 (10 pages).

[10] W.-T. Ni, A non-metric theory of gravity, Preprint of Dept. Phys. Montana State Univ. (December 1973) $25 \mathrm{pp}$.

[11] W.-T. Ni, Equivalence principle and electromagnetism, Phys. Rev. Lett. 38 (1977) 301-304.

[12] W.-T. Ni, Equivalence principles and precision experiments. In: Precision Measurement and Fundamental Constants II, B.N. Taylor and W.D. Phillips, eds. Nat. Bur. Stand. (US) Spec. Publ. 617, US Government Printing Office, Washington, DC (1984).

[13] W.-T. Ni, Probing the microscopic origin of gravity via precision polarization and spin experiments, Chin. Phys. Lett. 22 (2005) 33-35.

[14] A.P. Lightman and D.L. Lee, Restricted proof that the weak equivalence principle implies the Einstein equivalence principle, Phys. Rev. D8 (1973) 364-376.

[15] L. Blanchet, A class of nonmetric couplings to gravity, Phys. Rev. Lett. 69 (1992) 559-562.

[16] V.I. Denisov and M.I. Denisov, Verification of Einstein's principle of equivalence using a laser gyroscope in terrestrial conditions, Phys. Rev. D60 (1999) 047301 (4 pages). 
[17] Yu.N. Obukhov and G.F. Rubilar, Fresnel analysis of wave propagation in nonlinear electrodynamics, Phys. Rev. D66 (2002) 024042 (11 pages); arXiv.org/gr-qc/0204028.

[18] F.W. Hehl, Yu.N. Obukhov, G.F. Rubilar, and M. Blagojević, On the theory of the skewon field: from electrodynamics to gravity, Phys. Lett. A347 (2005) 14-24.

[19] F.W. Hehl and Yu.N. Obukhov, To consider the electromagnetic field as fundamental, and the metric only as a subsidiary field, Found. Phys. 35 (2005) 2007-2025.

[20] Y. Itin, A generalized photon propagator, preprint (May, 2007) 4 pp.; arXiv: 0705.0756 [hepth].

[21] V. Perlick, On the hyperbolicity of Maxwell's equations with a local constitutive law, preprint (March, 2007) 14 pp.

[22] S.M. Carroll, G.B. Field, and R. Jackiw, Limits on a Lorentz- and parity-violating modification of electrodynamics, Phys. Rev. D41 (1990) 1231-1240.

[23] Y. Itin, Caroll-Field-Jackiw electrodynamics in the pre-metric framework, Phys. Rev. D70 (2004) 025012 (6 pages); arXiv.org/hep-th/0403023.

[24] Yu.N. Obukhov and F.W. Hehl, On possible skewon effects on light propagation, Phys. Rev. D70 (2004) 125015 (14 pages).

[25] C. Lämmerzahl and F.W. Hehl, Riemannian light cone from vanishing birefringence in premetric vacuum electrodynamics, Phys. Rev. D70 (2004) 105022 (7 pages).

[26] C. Lämmerzahl, A. Macias, and H. Müller, Lorentz invariance violation and charge (non)conservation: A general theoretical frame for extensions of the Maxwell equations, Phys. Rev. D71 (2005) 025007 (15 pages).

[27] M. Haugan and C. Lämmerzahl, On the experimental foundations of the Maxwell equations, Ann. Phys. (Leipzig) 9 (Special Issue, 2000) SI-119-SI-124; arXiv.org/gr-qc/0002075.

[28] V.A. Kostelecky and M. Mewes, Cosmological constraints on Lorentz violation in electrodynamics, Phys. Rev. Lett. 87 (2001) 251304; V.A. Kostelecky and M. Mewes, Signals for Lorentz violation in electrodynamics, Phys. Rev. D66 (2002) 056005; V.A. Kostelecky and A.G.M. Pickering, Vacuum photon splitting in Lorentz violating quantum electrodynamics, Phys. Rev. Lett. 91 (2003) 031801; V.A. Kostelecky and M. Mewes, Sensitive polarimetric search for relativity violations in gamma-ray bursts, Phys. Rev. Lett. 97 (2006) 140401.

[29] P. Sikivie, ed., Axions '98. Proceedings of the 5th IFT Workshop on Axions, Gainesville, Florida, USA. Nucl. Phys. B (Proc. Suppl.) 72 (1999) 1-240. 
[30] F. Wilczek, Problem of strong P and T invariance in the presence of instantons, Phys. Rev. Lett. 40 (1978) 279-282.

[31] F. Wilczek, Two applications of axion electrodynamics, Phys. Rev. Lett. 58 (1987) 1799-1802.

[32] A. Lue, L. Wang, and M. Kamionkowski, Cosmological signature of new parity-violating interactions, Phys. Rev. Lett. 83 (1999) 1506-1509.

[33] P. Majumdar and S. SenGupta, Parity violating gravitational coupling of electromagnetic fields, Class. Quantum Grav. 16 (1999) L89-L94.

[34] W.-T. Ni, S.-S. Pan, H.-C. Yeh, L.-S. Hou, and J.-L. Wan, Search for an axionlike spin coupling using a paramagnetic salt with a dc SQUID, Phys. Rev. Lett. 82 (1999) 2439-2442.

[35] L. Cooper and G.E. Stedman, Axion detection by ring lasers, Phys. Lett. B357 (1995) 464-468.

[36] E. Zavattini, et al, Experimental observation of optical rotation generated in vacuum by a magnetic field, Phys. Rev. Lett. 96 (2006) 110406

[37] S. Biswas and K. Melnikov, Rotation of a magnetic field does not impact vacuum birefringence, Phys. Rev. D75 (2007) 053003 (4 pages).

[38] S.L. Adler, Vacuum birefringence in a rotating magnetic field, J. Phys. A40 (2007) F143-F152.

[39] A. Peres, Electromagnetism, geometry, and the equivalence principle, Ann. Phys. (NY) 19 (1962) 279-286.

[40] Yu.N. Obukhov and F.W. Hehl, Measuring a piecewise constant axion field in classical electrodynamics, Phys. Lett. A341 (2005) 357-365. 